\title{
Stakeholder Analysis of a Platform and Ecosystem for Open Innovation in SMEs
}

\author{
Jan Zibuschka, Uwe Laufs, and Wolf Engelbach \\ Fraunhofer IAO, \\ Nobelstr. 12, \\ 70569 Stuttgart, Germany \\ firstname. lastname@iao.fraunhofer.de
}

\begin{abstract}
In today's globalized economy, innovations become more and more crucial for the success of enterprises on the global markets. This article presents a reference architecture for an intermediary platform - and surrounding ecosystem - supporting the Open Innovation process in a network of SMEs and related stakeholders. Its main aim is to foster stakeholder dialogue in networked innovation management, but it also offers technical measures for data acquisition. We demonstrate business viability of the platform based on an analysis of involved stakeholders and their requirements.
\end{abstract}

Keywords: Intermediation, Open Innovation, Stakeholder Analysis, Platform.

\section{Introduction}

Innovations are becoming more and more crucial to the success of an enterprise due to the increasing competitiveness of the globalised economy. The development of new technologies, products and services offers an effective means to differentiate against competitors. However, to avoid leapfrogging and imitation, innovative enterprises need to be agile and flexible in bringing innovations to the market [1]. In the EU, SMEs' contribution to employment growth between 2002 and 2007 (84\%) has been much larger than could be expected from their share in total employment (67\%) [2]. Open Innovation [3] has been presented as a key paradigm to achieve a more agile innovation process. However, while the adoption of Open Innovation practices in SMEs is growing, it is much lower than in larger enterprises [4], depriving them of a central strategy for increased growth and competitiveness.

We aim to support SMEs by offering a platform enabling stakeholder dialogue and helping identify scenarios where coopetition or value network approaches involving several SMEs can lead to sustainable win-win situations [5]. In addition to innovative SMEs, the platform also addresses investors and innovation integrators.

The following section will give related work. Section 3 presents the overall approach and architecture of the platform, followed by a stakeholder analysis showing elicited requirements and how they are met by the platform design in section 4 . Section 5 discusses advantages of our proposed architecture. 


\section{Related Work}

There have been several papers investigating the deployment of Web 2.0 technologies in the context of innovative SMEs and SME networks, such as the ones by Lindermann et al [6] as well as Blinn et al [7]. Those papers offer a requirements analysis and first steps in a design science approach, but no resulting artifact has been presented yet. Duin et al [8] present components for such a system, but do not integrate them or discuss their business value. We are not the first to use ontologies in innovation management; similar approaches are presented by Li et al [9].

We use a combination of social and semantic web to mitigate the chicken-egg effect. This is discussed in much more detail in [10]; however, we introduce it to innovation management and illustrate its value in this use case.

While existing approaches focus either on the participating SMEs, and supporting communications between them, capturing the knowledge of the non-executive workers, or capturing the users' knowledge, our approach integrates a wider set of stakeholders, discusses their requirements and presents a fitting reference architecture.

\section{Architecture}

Intermediaries in open innovation settings have several advantages, specifically for SMEs [11]. They have the potential to aggregate common and field-specific knowledge bases, as well as acceleration and standardization of innovation-related information exchange, and support in the formation of temporary virtual organizations [12]. Additionally, the hosted application service provider paradigm also shared by our web based intermediary platform is the most viable means of engaging SMEs in complex e-business activities in the area of innovation [13].

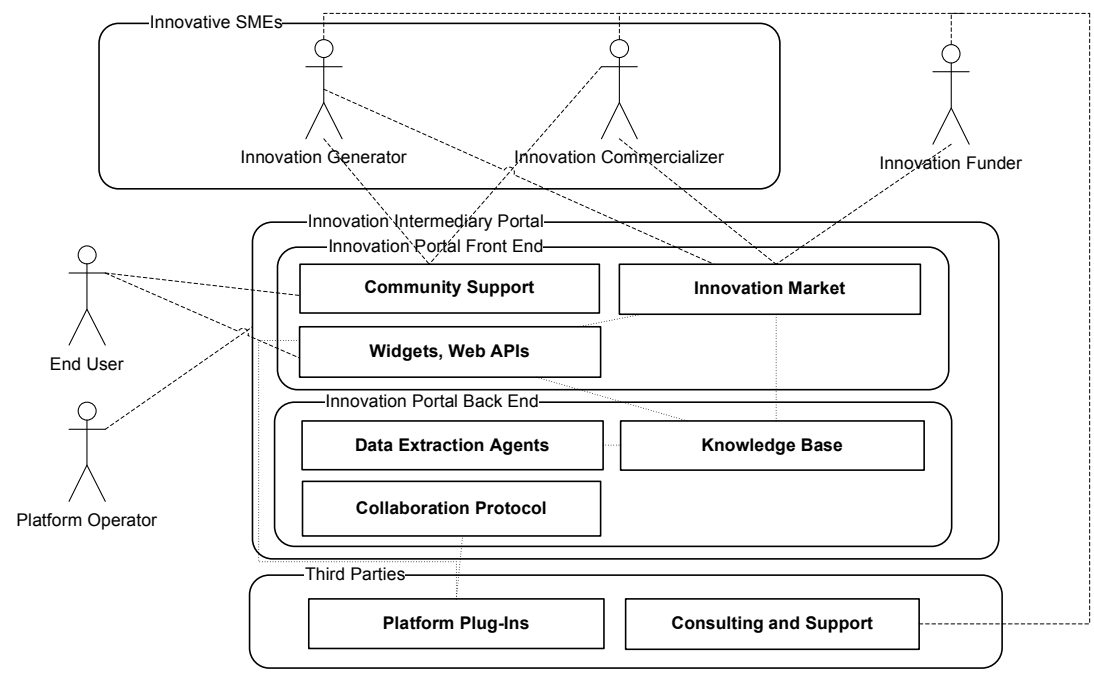

Fig. 1. Overview of System Structure and Stakeholders 
An overview of the overall proposed architecture, data flows (dotted lines) and stakeholder involvement in use cases (dashed lines) is given in Figure 1. The main components of the envisioned core innovation intermediary platform are:

- A community portal, acting as the entry point, hosting community support services, such as discussion boards.

- A set of agents extracting information from available external data sources on the WWW or semantic web, such as software agents extracting information from publication databases, technology blogs, or semantic services on the internet of knowledge.

- Semantic Knowledge Base aggregating information from agents, user feedback, experiences from previous projects and data mining. This data will be the base for e.g. technology forecasting and decision support in different phases of networkcentric innovation processes.

- Widgets and Web APIs around innovation management, such as feedback mechanisms for new or emerging products or innovative enterprises. Those components are the main source of input to the system besides the agents, are often based on earlier agent input, and provide feedback from users to fine-tune the system. Offering tools to users to motivate them to contribute in open innovation processes is discussed in some detail in [14].

- A collaboration protocol definition allowing collaboration tools implementing it to be able to become part of a temporary network of businesses or individuals within or beyond the platform. The portal-mode is aimed at small SMEs, leveraging a Software as a Service approach to lower the barriers they are facing when entering enterprise collaboration systems [13].

- The Web APIs, along with the collaboration protocol, form the basis for the extensible plug-in system enabling an ecosystem of third party plug-ins that may be integrated in the platform.

\section{Stakeholder Analysis}

Stakeholder analysis is widely used as a tool for gathering and evaluating comprehensive requirements in information system design [15]. The stakeholder analysis presented here is the result of interviews with representatives of all the identified stakeholder groups involved in the platform. It includes feedback from several SME, research institute and large industry partners commonly involved in Open Innovation processes. We followed the research paradigm of design science, seeing the design of the platform as a search process, which we steered be eliciting stakeholder feedback on designs early on. Several iterations of the design were presented to the stakeholders, and their feedback integrated. The design presented here can be seen as the core, which holds the main business value, and prevailed across all application fields.

We mainly base on stakeholder roles identified in [3], but also including users (customers, lead users and domain experts from the broader web population), as well as the platform operator of the intermediary design presented here, leading to the following roles: Innovation Commercializer, Innovation Funder, Innovation 
Generator, End User, and Platform Operator. We note that roles are not exclusive, for example it is very viable that the platform operator is as well an Innovation Architect and an Innovation Commercializer. This is in line with the definition of the roles given in [3].

For our analysis, we use the stakeholder requirements dimensions identified by Kafenzis et al in their analysis of knowledge marketplaces [16], namely: A viable revenue model for the involved stakeholders, mitigation of the chicken-egg problem of getting all stakeholder groups to adopt the multi-sided platform at the same time, and risk/trust requirements related to the disclosure of potentially valuable intellectual property in the platform.

Each of these requirements dimensions will be discussed for every stakeholder, illustrating how concerns are addressed and value is created within the platform.

\subsection{Innovation Commercializers}

Innovation Commercializers are defined as innovation marketers or one-stop centres in [3]. Their main role is to act as middlemen in the commercialization of innovation, mediating between end users and innovation generators.

- Revenue Model: Innovation Commercializers benefit from participation in the platform by being able to pick and match innovation, while also having the opportunity to evaluate potentials of technologies due to the user feedback aggregated within the platform.

- Chicken-Egg Problems: Innovation Commercializers will be able to directly benefit from the platform, even if no innovation explorers are present, due to the semantic agents aggregating information on innovations from the web, providing information relevant in the commercialization process, such as extracted capabilities of innovative technologies.

- Risk/Trust: Innovation Commercializers need to make sure that the technologies or products the innovation generators are supplying meet their requirements. This is usually realized in the form of service level agreements, contractual obligations negotiated between the parties.

\subsection{Innovation Generators}

Innovation Generators are defined as innovation explorers, merchants, architects or missionaries in [3]. Their role is to provide innovations, and they are one of the main sources of innovations processed within the platform.

- Revenue Model: Innovation Generators can benefit from using the platform to identify uses for their innovations and commercialize them in cooperation with Innovation Commerzializers and Funders. Explorers and merchants are mainly supplying their innovative solutions, while architects can offer services to identify and integrate innovations made available to them by other participating enterprises via the platform. Missionaries can use the platform to influence product development to more closely match their vision. 
- Chicken-Egg Problems: Innovation Generators are not dependent on other parties to benefit from the platform, as the coordination the platform offers between innovation generators in different fields and between explorers and architects creates value through product and service innovation. Depending on the quality of available information in the specific innovation domain, a certain amount of other interested users or own configuration will be necessary to leverage the platform.

- Risk/Trust: Innovation Generators need to protect their innovative intellectual property, and thus cannot accept an unregulated presentation of their ideas on the platform. The platform will offer processes that disclose relevant innovation generators to interested parties, but does not disclose the relevant innovations.

\subsection{Innovation Funders}

Are defined in [3] as either innovation investors or benefactors. They provide funding for innovative project, enabling other platform participants to e.g. spin out their innovations or acquire additional venture capital.

- Revenue Model: Innovation funders benefit from participation in the platform due to the combination of innovations with relevant user feedback supplied by the idea marketplace by being able to more precisely ex-ante valuate the innovations they are investing in.

- Chicken-Egg Problems: Innovation Funders will be able to directly benefit from the platform, even if no innovation explorers are present, due to the semantic agents aggregating information on innovations from the web, providing information that can already be used for technology forecasting and ex-ante evaluation of investments they may make outside of the platform.

- Risk/Trust: Of course, funding innovations is, as any venture funding, inherently risky. Beyond the aforementioned information, the platform itself can hardly mitigate this risk. It is expected that the platform will only be used for match-making between innovation funders and Innovation Generators or Commercializers, and not for e.g. an actual transfer of funds.

\subsection{End Users}

The main user groups that are expected to participate in the intermediary are early adopters/lead users of the relevant technologies providing feedback on their expectations and experiences, as well as domain experts offering their expertise in the ideas market.

- Revenue Model: End users are empowered to influence the development of new products and services. In addition, idea marketplaces often contain challenges where marketplace participants such as innovation commercializers offer rewards for the successful solving of specific problems. Also, innovation architects or commercializers may provide incentives such as coupons or early access to products to users providing valuable feedback.

- Chicken-Egg Problems: Attracting users is central to the success of the platform, and several measures have been taken to appeal to relevant user groups. The platform provides widgets that are directed at the users, helping them in visualizing 
innovation-relevant facts while at the same time collecting their feedback. This measure also is aimed at multiplicators like technology blogs, which may employ such a widget to e.g. host a survey considering a specific innovation.

- Risk/Trust: A user providing an idea may want to protect it against misuse, such as by an enterprise not paying for his idea and not using it officially, but still acting on it. While this problem cannot be easily solved technologically, the platform should not encourage such behaviour.

\subsection{Platform Operator}

The platform operator may be a SME in one of the Open Innovation-related roles from [3], or an independent entity, e.g. a provider of a commercial idea marketplace.

- Revenue Model: The Platform Operator has several possibilities to monetarize the platform. He may offer advanced premium services to paying customers. He may present advertising to and data-mine the non-paying customers, especially in the context of the widget toolkits, but also in the context of the internal innovation management process, which may in turn form the basis for further decision support services offered to premium users. He may also offer consultancy to third parties developing services for the ecosystem.

- Chicken-Egg Problems: The platform provider is aided in the typical chicken-egg problem by the semantic technology. Of course, he still needs to find interested parties. Promoting the tool in SME networks (via individualized deployments) and multiplicators [17] (via widgets) is essential. To this end, several implementations in SME networks are planned.

- Risk/Trust: The platform operator needs to be a trusted partner for the participating SMEs. It should be made very clear what data is mined (e.g. data related to assessment of innovations) and what data isn't (e.g. information from specific innovation projects). If the Platform Operator is an involved SME itself, it needs to be trusted by its partners, and may want to consider a more federated deployment of the platform.

\section{Conclusion}

We presented an innovation intermediary platform design meeting elicited stakeholder requirements. Building on this, we demonstrated viable incentives and revenue models for all participants using a stakeholder analysis based on interviews and stakeholder interactions with prototypical components. We addressed the chicken-egg problem often troubling multi-sided platforms by employing a combination of semantic technologies and stakeholder feedback, providing some information as a working basis right away, allowing for involvement of all stakeholders in the fine-tuning of aggregated information, and generally meeting business requirements of Open Innovation stakeholders, specifically in the areas of instant usefulness of the platform and revenue opportunities. 


\section{References}

1. Horii, R., Iwaisako, T.: Economic Growth with Imperfect Protection of Intellectual Property Rights. Journal of Economics 90, 45-85 (2007)

2. European Commission: First Section of the Annual Report on EU Small and Mediumsized Enterprises (2008)

3. Chesbrough, H.W.: The Era of Open Innovation. MIT Sloan management review 44, 35$41(2003)$

4. van de Vrande, V., de Jong, J.P., Vanhaverbeke, W., de Rochemont, M.: Open innovation in SMEs: Trends, motives and management challenges. Technovation 29, 423-437 (2009)

5. Hearn, G., Roodhouse, S., Blakey, J.: From value chain to value creating ecology. International Journal of Cultural Policy 13, 419-436 (2007)

6. Lindermann, N., Valcárcel, S., Schaarschmidt, M., von Kortzfleisch, H.: SME 2.0: Roadmap towards Web 2.0-Based Open Innovation in SME-Networks. In: Creativity and Innovation in Small and Medium-Sized Enterprises, pp. 28-41 (2009)

7. Blinn, N., Lindermann, N., Fäcks, K., Nüttgens, M.: Web 2.0 in SME Networks - A Design Science Approach Considering Multi-perspective Requirements. Value Creation in E-Business Management, 271-283 (2009).

8. Duin, H., Geven, A., Dittenberger, S., Tscheligi, M., Hesmer, A., Thoben, K.D.: A Toolset to Support the Early Stage of Innovation. Towards new challenges for Innovative management practices 2, 111-118 (2008)

9. Li, Y., Wang, J., Li, X., Zhao, W.: Design creativity in product innovation. The International Journal of Advanced Manufacturing Technology 33, 213-222 (2007)

10. Ankolekar, A., Krötzsch, M., Tran, T., Vrandecic, D.: The two cultures: Mashing up Web 2.0 and the Semantic Web. Web Semantics: Science, Services and Agents on the World Wide Web 6, 70-75 (2008)

11. Lichtenthaler, U., Ernst, H.: Intermediary Services in the Markets for Technology: Organizational Antecedents and Performance Consequences. Organization Studies 29, 1003-1035 (2008)

12. Carlsson, S., Corvello, V., Migliarese, P.: Enabling open innovation: Proposal of a framework supporting ICT and KMS implementation in web-based intermediaries. In: 17th European Conference on Information Systems, Verona, Italy, pp. 2218-2230 (2009)

13. Brown, D.H., Lockett, N.: E-business, innovation and SMEs: the significance of hosted services and firm aggregations. International Journal of Entrepreneurship and Innovation Management 7, 92-112 (2007)

14. von Hippel, E., Katz, R.: Shifting Innovation to Users via Toolkits. Management Science 48, 821-833 (2002)

15. Pouloudi, A.: Aspects of the stakeholder concept and their implications for information systems development. In: Proceedings of the 32nd Annual Hawaii International Conference on System Sciences, 1999, HICSS-32, p. 7030 (1999)

16. Kafentzis, K., Apostolou, D., Mentzas, G., Georgolios, P.: A Framework for Analysis and a Review of Knowledge Asset Marketplaces. Practical Aspects of Knowledge Management, 301-313 (2002)

17. Goldenberg, J., Han, S., Lehmann, D.R., Hong, J.W.: The Role of Hubs in the Adoption Process. Journal of Marketing 73, 1-13 (2009) 